SAND75-0248

Unlimited Release

UC-71

\title{
Transportation Environment Data Bank Index
}

\author{
C. A. Davidson, J. T. Foley
}

Prepared by Sandia Laboratories, Albuquerque, New Mexico 87115

and Livermore, California 94550 for the United States Energy Research

and Development Administration under Contract AT (29-1).789

Printed June 1975 
Issued by Sandia Laboratories, operated for the United States Energy Research and Development Administration by Sandia Corporation.

\section{NOTICE}

This report was prepared as an account of work sponsored by the United States Government. Neither the United States nor the United States Energy Research and Development Administration, nor any of their employees, nor any of their contractors, subcontractors, or their employees, makes any warranty, express or implied, or assumes any legal liability or responsibility for the accuracy, completeness or usefulness of any information, apparatus, product or process disclosed, or represents that its use would not infringe privately owned rights. 


\section{DISCLAIMER}

This report was prepared as an account of work sponsored by an agency of the United States Government. Neither the United States Government nor any agency Thereof, nor any of their employees, makes any warranty, express or implied, or assumes any legal liability or responsibility for the accuracy, completeness, or usefulness of any information, apparatus, product, or process disclosed, or represents that its use would not infringe privately owned rights. Reference herein to any specific commercial product, process, or service by trade name, trademark, manufacturer, or otherwise does not necessarily constitute or imply its endorsement, recommendation, or favoring by the United States Government or any agency thereof. The views and opinions of authors expressed herein do not necessarily state or reflect those of the United States Government or any agency thereof. 


\section{DISCLAIMER}

Portions of this document may be illegible in electronic image products. Images are produced from the best available original document. 
SA ND 75-0248

Unlimited Release

Printed June 1975

$\mathrm{UC}-71$

TRANSPORTATION ENVIRONMENT DATA BANK INDEX

\author{
C. A. Davidson \\ J. T. Foley \\ Applied Mechanics Division II 1542 \\ Sandia Laboratories \\ Albuquerque, NM 87115
}

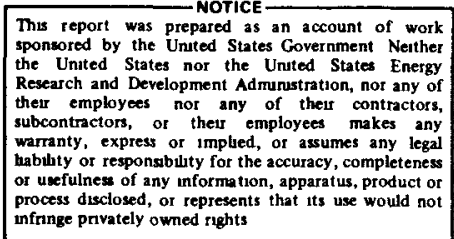

infringe privately owned rights

\begin{abstract}
In an effort to determine the environment intensities to which shipping containers will be exposed, a "Data Bank" of environmental information has been established by Sandia Laboratories, Division 1542, for the ERDA Division of Waste Management and Transportation. This document is an index which can be used to request data of interest.
\end{abstract}

Printed in the United States of America

Avallable from

National Technical Information Service

U S Department of Commerce

5285 Port Royal Road

Springfield, Virginia 22151

Price Printed Copy $\$ 4.00$ Mict of tiche $\$ 2.25$ 
$\bullet$$$
\text { ' }
$$ 


\section{CONTENTS}

$\underline{\text { Page }}$

Introduction

The Environments

The Data

10

Mechanics of the Data Bank

Data Sources

Avallability of the Data

The Index

Normal Environments

Abnormal Environments 


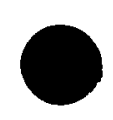


TRANSPORTATION ENVIRONMENT DATA BANK INDEX

Introduction

The purpose of the Transportation Environment Data Bank is to store and make avallable descriptions of the environment of transportation expressed in engineering terms. The data are intended to provide environmental criteria to shipping container design and test engineers as a basis for their functions. The Transportation Environment Data Bank is a segment of a larger ERDA/DOD environmental Data Bank for weapons application.

For purposes of indexing and data retrieval, the data are catalogued under two major head1ngs: Normal and Abnormal Environments. The diagram below illustrates the relationship between these environmental designations.

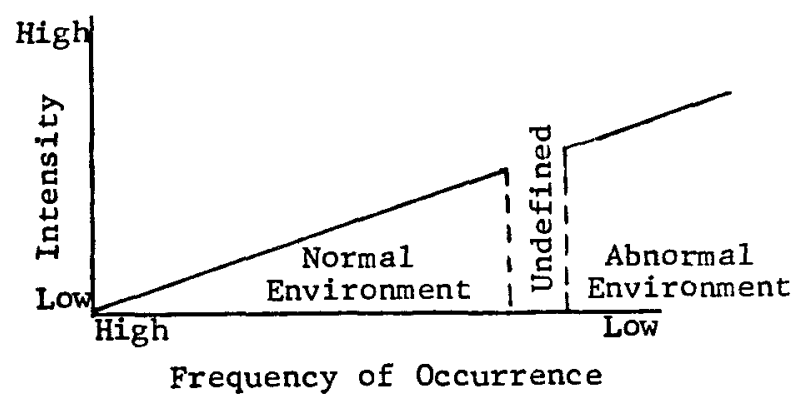

Normal environments are those which will be encountered at some level during every shipment. They have a high frequency of occurrence, but a relatively low intensity. The abnormal or accident environments, on the other hand, are characterized by higher intensities but lower frequency of occurrence. The very high intensities of environment occur very infrequently. The abnormal environments, while often called by different names to differentiate them, have actually the same engineering parameters as the normal. Thus, an acceleration is experienced by cargo as a truck crosses rallroad tracks at $50 \mathrm{mph}$. A greater acceleration may result if it strikes a bridge abutment at the same speed. To make the difference clear in a succinct manner, the latter is termed, "1mpact." These short descriptions are shown in the index in parentheses where they apply.

The Environments

Twelve categories of environment have been identıfied as relevant to transportation. These include: 

1. Acceleration/Time *
2. Acoustic Nolse
3. Atmospheric Contents
4. Fragmentation ${ }^{*}$
5. Humidity
6. Precipitation
7. Pressure
8. Radiatıon ${ }^{*}$
9. Shock
10. Temperature*
11. Vibration
12. Wind ${ }^{*}$

Three factors operate to $11 \mathrm{~m} 1 \mathrm{t}$ the number of these environments for which abnormal levels require consideration:

1. Some environments reach an absolute limit regularly, and the limit thus is withın the normal range;

2. The abnormal aspects of some of them are encountered so infrequently as to be of little interest; and

3. Protection against the effects of some environments include protection against the abnormal.

After eliminating from consideration those listed environments falling within the above limitations, only the environments indicated by an asterisk in the above list are of interest in the abnormal considerations. Figure 1 presents the environments which relate to transportation and the detalls of the abnormal environments as to input and response.

Input may be defined as the environmental level which exists at the cargo interface, while response is considered as the way in which the cargo reacts to the environments. As an example, consider the pressure environment. Pressure has both normal and abnormal levels. The normal, which all cargo will experience, includes the variations in at mospheric pressure at a specific location, as well as the variations produced by changes in elevation, both on the earth's surface and whlle flying. The abnormal aspects of the pressure environment are the result of exposure to an explosion or to immersion (inputs); if the intensity level of input is sufficiently high, a crush response of the cargo will result.

Four of the environments are considered only in the normal aspects for reasons mentioned above. One environment, fragmentation, is considered only in the abnormal aspect. This is be cause no elements which will cause puncture to the cargo are present under normal conditions, 1.e., will be encountered frequently during transportation.

\footnotetext{
*Environments with both normal and abnormal aspects.
} 


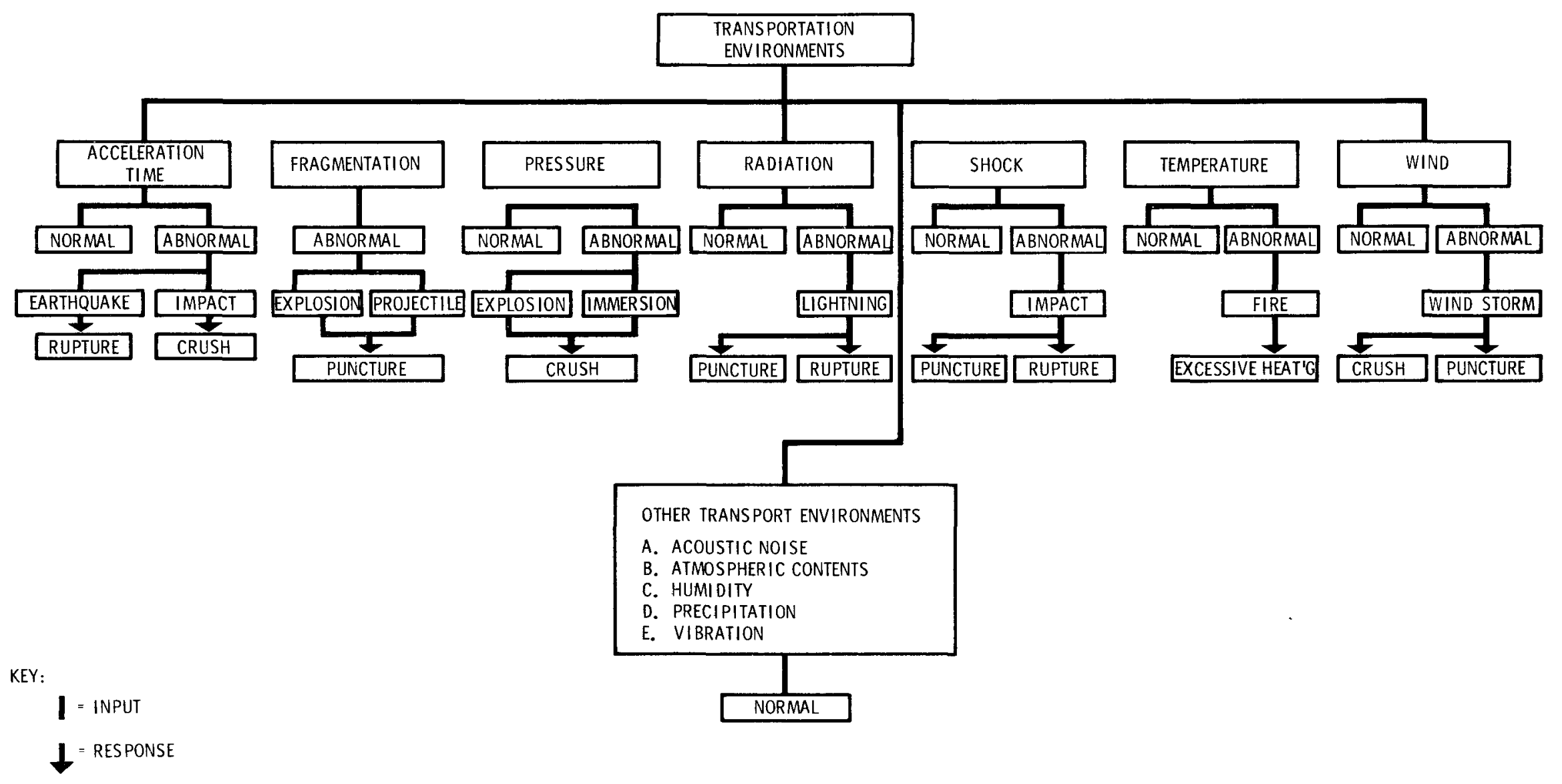

Figure 1. Structure of the Transportation Environment Data Bank 
The Data

Both input and response data contained in the Transportatıon Environment Data Bank relatıng to the normal environments are measured data. They were obtained from reliable sources, both withın Sandia Laboratories and from other reputable agencies. When data were required, but not avallable, the Data Bank operators arranged for measurements to provide the information.

Most input data in the Data Bank under the heading of Abnormal Environments, however, were not measured directly. Because of the number and randomness of occurrence and type, it is $1 \mathrm{mpos}-$ sible to provide engineering instrumentation for measurement. If an instrumented vehicle is subjected to a staged accident, it represents only the one type of event. Therefore, it is necessary to use statistical techniques to characterize the range of environmental intensities which may be experienced by cargo in accidents. The data to which these techniques are applied are often estimated. The basic data which are most useful to estımate abnormal inputs include the following.

Abnormal Input

1. Earthquake

2. Impact

3. Explosion

4. Projectile/Impalement

5. Immersion

6. Lightning

7. Fire

8. Windstorm
Useful Data Parameters

Time History

Velocity

Pressure or Missile Velocity, Radius

Velocity, Radius

Depth

Voltage, Amperage, Rise Time, etc. Temperature or Heat Flux and Duration

Velocity

It is these types of data, or information which permit the derivation of these values, which are stored in the input section of the Abnormal Environment part of the Transportation Environment Data Bank. The response section of the Bank is expressed in terms of damage to cargo. Since the damage can be described and measured, measurements and descriptions are included wherever possible.

Mechanics of the Data Bank

The data which have been retrieved and entered in the Data Bank are on microfilm. Two forms of microfilm are used: aperture cards and microfiche. Facilities for viewing the data and obtainıng hard copy are located at the bank site. When processıng large requests for information, the Bank's operators have access to automated means for obtainıng hard copy.

When either raw data or published information is acquired, it is reviewed for engineering data content. Pertinent information is then extracted, collated, and microfilmed. The data cards and/ or microfiche are filed in numerical order without regard to subject. This simple technique is 
possible because of a computerızed indexing and retrieval program which was developed for eff 1 clent use of the Data Bank.

The use of a computer-aided system makes it possible to obtain a more flexible file, permits more complete data retrieval, shortens the time necessary for search, and makes feasible more frequent publication of a current index.

\section{Data Sources}

Normal environmental information for inclusion in the Data Bank is acquired by the Data Bank's operators from many sources. Miltary agencies, such as Aberdeen Proving Ground, Wright Air Development Center, Naval Ordnance Test Center, and Army QM R\&E Center, and industrial groups, such as ralloads, aircraft companies, and trucking concerns, have contributed information. A considerable portion of the information has been obtained from the various test groups in Sandia Laboratories. The Data Bank's operators not only extract information from published sources, but engage in specific research activities to obtain data not otherwise avallable.

Abnormal environmental information is obtained primarily from U. S. Department of Transportation sources and reports produced by the National Transportation Safety Board. Contacts have been established with various individuals and agencies responsible for accident investigation in the various modes of transportation. An information sources file is maintained as an adjunct to the Bank which integrates this Data Bank's efforts with other Data Banks collecting related accident information such as accident rates, fatalıtıes, etc.

\section{Avallability of the Data}

The information in the Data Bank is avallable, within the limits of time, workload, and security regulations, to any requestor. Requests for data should be sent to Division 1542, Sandia Laboratories, Albuquerque, New Mexico, 87115. The request should either outline the specific area for which information is desired, or it should list specific entries, including serial numbers. Requests will be processed as time allows. Agencies outside of the ERDA complex will be furnished information subject to the review and release procedures established by the ERDA. 
The index provided on the following pages is what the Bank's operators call a category index. Entry is made into this index by finding the environmental category of interest, such as shock, temperature, vibration, etc. The 12 categories are listed alphabetically.

The two-part number listed after the title of a particular Data Bank entry is the access number for the data on flle. The first number, e.g., 1614, 1s the flle number. The number after the dash (-) is the number of file cards avallable on the subject. Requests for hard copies of data should cite these two numbers. 


\section{Normal Environments}




\begin{tabular}{|c|c|c|}
\hline $\begin{array}{l}\text { LUADS IMPCSED ON CARGC BY FLIGHT ANC LANOING } \\
\text { MANFUVER LOAD DATA C-130 AIRCRAFT } \\
\text { FATIGUE DAMAGE PARAMETERS, TRANSPORT AIPCRAFT } \\
\text { GUST LOAD FRFQUENCY, AIRCFAFT FLIGHTS, } 4 \text { - } 45 \text { KILCIFEET, IN STCRM } \\
\text { AIRCRAFT RECDRDINGS DURING A TURBULENCE INCIDENT }\end{array}$ & $\begin{array}{l}\text { UNC } \\
\text { UNC } \\
\text { UNC } \\
\text { UNC } \\
\text { UNC }\end{array}$ & $\begin{array}{l}00750-001+ \\
00944-002+ \\
01085-001+ \\
01087-002+ \\
01090-001+\end{array}$ \\
\hline - ACCELERATION/TIME & & \\
\hline $\begin{array}{l}\text { ACCELERATION, SHIP MOTIONS, MAXIMUM } \\
\text { ACCELERATIONS ON PACKAGES STRREL IN SHIPS HULDS } \\
\text { ACCELERATION, EXPECTED PEAK VALUES, NARINE } \\
\text { SHIP MOTIONS, SS SILVER MAHINFR, NEW YORK-BRENEFHAVEN, GERMANY } \\
\text { SHIP MOTIONS, T-2 TANKER, ESSO ASHVILLE,NORMAL TPERATIONS } \\
\text { WAVE HEIGHT OISTRIBUTION - ATLANTIC COAST }\end{array}$ & $\begin{array}{l}\text { UNC } \\
\text { UNC } \\
\text { UNC } \\
\text { UNC } \\
\text { UNC } \\
\text { UNC }\end{array}$ & $\begin{array}{l}00739-001+ \\
00740-001+ \\
00741-001+ \\
00746-001+ \\
00747-001+ \\
01573-004+\end{array}$ \\
\hline
\end{tabular}

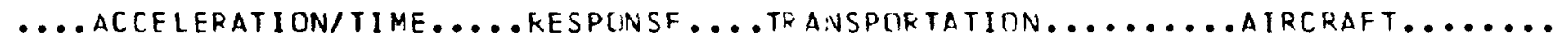

GUST LOAD FREQUENCY, AIRCRAFT FLIGHTS,40-45KILOFFFT, IA STORM UNC OLO87-002+ AIRCRAFT RECOROINGS DUKING A TURGULFNCF INCIDFNT 


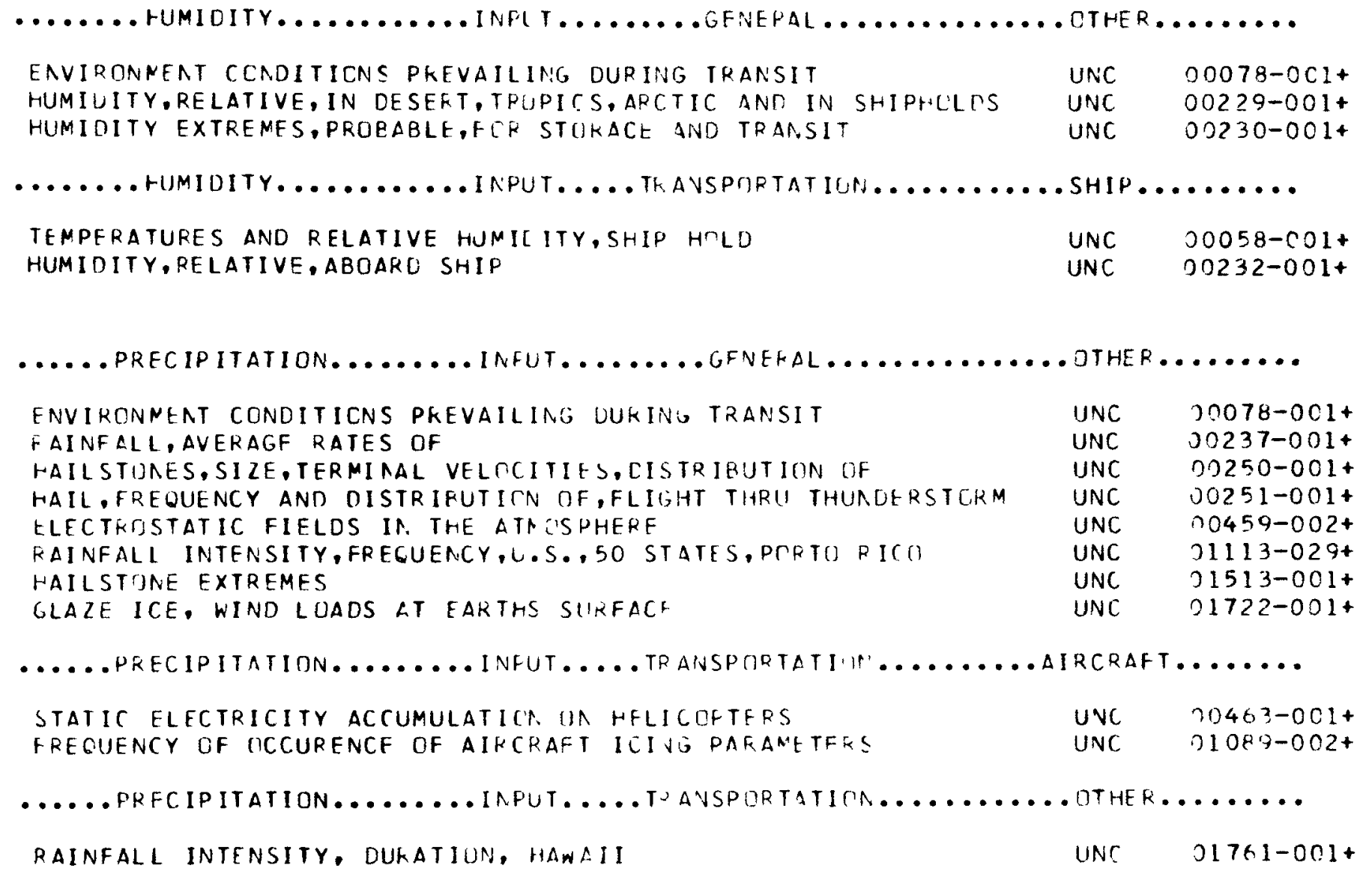




\begin{tabular}{|c|c|c|c|c|c|c|}
\hline \multicolumn{2}{|c|}{ PRESSURE, TYPICAL } & AL DAILY VS & TIME & OF DAY & UNC & $00140-001+$ \\
\hline BAROMETP & IC VALU & JES. MAXIMUM & NORMA & I VARIATION, UNITED STATES & UNC & $00143-0014$ \\
\hline TYPHOON & REPORT & - PACIFIC & CCEAN & -1959 & UNC & $01802-003+$ \\
\hline TYPHOON & REPORT & - PACIFIC & CCEAN & -1960 & UNC & $01803-0034$ \\
\hline TYPHOON & REPQRT & - PACIFIC & CCEAN & -1961 & UNC & $01804-0034$ \\
\hline TYPHOON & REPORT & - PACIFIC I & OCEAN & -1962 & UNC & $01805-004 t$ \\
\hline TYPHOON & REPORT & - PACIFIC & OCEAN & -1963 & UNC & $01806-003+$ \\
\hline TYPHOON & REPORT & - PACIFIC & OCEAN & -1965 & UNC & $01807-003+$ \\
\hline TYPHODN & REPORT & - PACIFIC & OCEAN & -1966 & UNC & $01808-003+$ \\
\hline TYPHOON & REPORT & - PACIFIC & OCEAN & -1967 & UNC & $01809-003+$ \\
\hline TYPHOON & REPORT & - PACIFIC & OCEAN & -1968 & UNC & $01810-004 t$ \\
\hline TYPHOON & REPORT & - PACIFIC & CCEAN & -1969 & UNC & $01811-002+$ \\
\hline TYPHOON & REPORT & - PACIFIC & OCEAN & -1970 & UNC & $01812-003+$ \\
\hline TYPHOON & REPORT & - PACIFIC & OCEAN & -1974 & UNC & $01813-003+$ \\
\hline
\end{tabular}

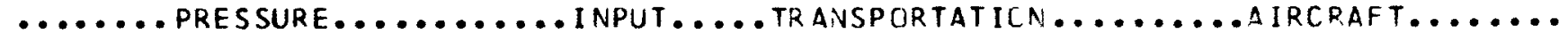

PRESSURE CHANGES, ATMOSPHEFIC, AIRCRAFT CARGO HOLDS UNC O0147-001+ PERFORMANCE AND LOADING-C130B UNC $00143-001+$ PERFORMANCE AND LOADING-C133A UNC $00149-001+$ FLIGHT PROFILE,NORMAL,C133 CARGO AIRCRAFT UNC OO150-001+ FLIGHT PROFILE, NDRMAL,C124 CARGD AIRCRAFT UNC D0151-001+

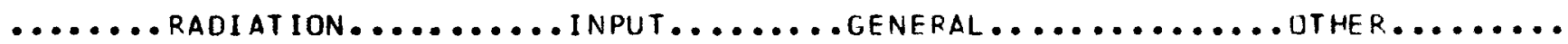

ENVIRONMEAT CONDITICNS PREVAILING CURING TRANSIT TEMPERATURE, EXTREMES/OURATION,FOR STORAGE,HANDL ING, TPANSPORT UNC $00080-002+$ ELECTROSTATIC FIELOS IN THE ATMCSPHERE STATIC ELECTRICITY-ELECTRICAL CHARACTERISTICS OF ATMOSPHERE UNC O9460-002+ RADIATICN,SOLAR, AT EARTH S SURFACE AND ATMOSPHERE S EDGE UNC OO479-OO3+ RADIATICN DATA.SOLAR RADIATICN,MEAN DAILY TOTAL,FLAT SURFACE, LOCATIONS, US/CANADA UNC OO495-004+ INSOLATION, MONTHLY MAPS OF MEAN DAILY,FOR THE UNITEO STATFS UNC OO831-006+ LIGHTNING CHARACTERISTICS

UNC 01107-009+

$\ldots \ldots . .$. RADI ATION......... INPLT..... TRANSPORTATIGN........ IRCRAFT......

STATIC ELECTRICITY ACCUMULATICN OA HELICCPTERS

HEL ICOPTER STATIC ELECTRICITY MEASUREMENTS

UNC $\quad 00463-001+$

UNC $\quad 00503-003+$

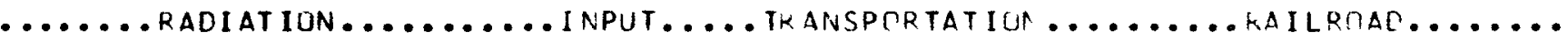

TEMPERATURE, INTERNAL AIR, RAILROAD BCXCAR IN DFSFRT ONC OOOE-OCI+

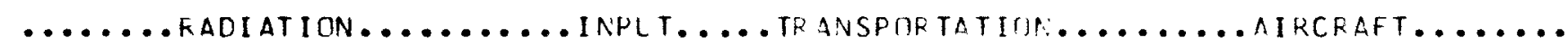


SHOCK MFASUREMENTS, COCKSIDE/SHIPBOARE HANDLINC PACKAGE DROPPED ON FARTH, WOUD ANO CONCPETE FLOIIPS

UNC

UNC

UNC

UNC.

UNC

UNC

UNC

UNC

UNC
$20335-001+$ $00337-001+$ $07243-0 \mathrm{Cl}+$ $20814-001+$ $21744-0 \mathrm{Cl}+$ $01745-047+$ $01746-057+$ $01749-058+$ $01752-041+$

......... SHOCK............ INPUT .....TR.ANSPURTATILA........AIRCRAFT......

DYNAMIC ENVIKCNMENT, TUKBGJET CARGC AIRCRAFT

SHOCK DATA.AIRCRAFT/CARGO/

SHUCK ZCNES REGISTEREC IN AIR CARGC TESTS DURING FLICHT

UNC A1533-0C2+ SHOCK. C5A CARGO FLODR, (SEE 1439)

$\begin{array}{ll}\text { UNC } & 30334-001+ \\ \text { UNC } & 00336-001+ \\ \text { UNC } & 01444-042+\end{array}$

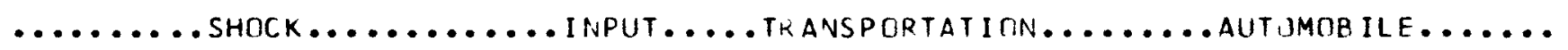

SHOCK DATA-TRUCKS

SHOCKS FROM ROAD IRRECULARITIES AND TCWBAR SNATCH

UNC $\quad 00311-001+$

ENVIRTNMENT IN VEHICLES TRAVELING OVER PAVED RCACS

UNC $\quad 00314-001+$

UNC $20315-001+$

UNC 00317-001t

VIBRATIGN-ACCELEKATIONS IN HIGHWAY OPEZATION CF TRUCKS

SHOCK 2 CNES REGISTEREC INCARGC DURING SURFACE TRANSFER

DYNAMIC ENVIRONMENT ON A FLAT BED TRACTOR/TPAILFR (AFC/LUL')

UNC

$00370-001+$

FINAL ANALYSIS,AEC/DOD FLATBFD TRUCK TFST

VIBKATION/SHOCK, $21 / 2$ TON TRUCK

VIBKATICN/SHOCK AIR SUSPENSITA VAR

SHOCK/VIBPATION SUMMARY, UNION CARPIDE TRUCK TFST

UNC.

$10813-001+$

$01006-008+$

UNC $01083-041+$

UNC $11264-008+$

UNC $01277-006+$

UNC $\quad 01312-007+$ SHOCK DATA.UNICN CARBIDE TRUCK TEST

UNC

$1314-013+$

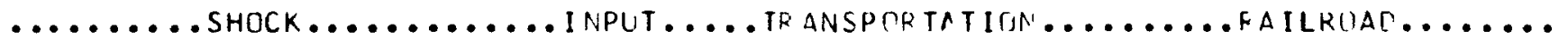

FAIL HUMP SHUCK CRITERIA SUMMAKY

UNC

BOXCAR SHOCK CRITERIA SUMMARY

ATMX CAR SHOCK CRITERIA SUMMAKY

SHOLK IVIBRATION ENVIRCNMENT SLIMMARY, RAIL TRANSPCKT

UNC

SHUCK SPFCIRA SUMMARY, SIX-ATMX CAR TRAIN

SPEEDS. SWITCHING.OBSERVED AT VARIOUS HIJMP YAKCS

SPECDS, SWITCHING, OBSERVATIUN OF.AT ILLINJIS CINTKAL FF YAFDS

SPEEDS, SWITCHING, DBSERVED

SHCCK, SKITCHING, RAIL, CCCUFANCE IF

SPFED, SWITCHING.DATA COMPARFD WITH NEW UATA, NY CFNTHAL RI

SPEED. HUMPING, FREOUENCY OF INPACTS AT VAYITUS

RAIL SWITCHING AND HUMPING OPERATIENS

$31203-002+$

B1210-002+

B1320-011+

C1215-012+

33 ? $84-001+$

30285-024t

J0?86-001+

J0287-CO1+

Jn2ks-0rit

J0) $4.4-001+$

วI) 2 on-nn?t 


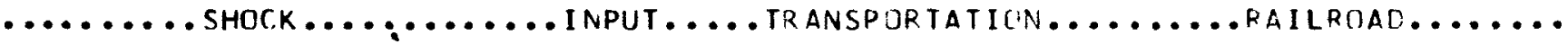

SHOCK DATA,RAILROAD SWITCHING

SHOCK DATA,RAILRDAD SWITCHING

UNC $\quad 30293-002+$

$00294-002+$

SHDCK DATA, RAILROAD SWITCHING

$00295-002+$

SHOCK DATA.RAIL/BOXCAR/

SHOCKS IN TRAINS WHEN STOPPING, STARTING AND SWITCHING

UNC

UNC

UNC

$00297-001+$

RAILROAD SWITCHING TEST OF SPFCIAL RAIL CAR ATMX 500

UNC

$00300-001+$

RAIL HUMPING TEST, 500 SERIES ATMX CAR

UNC $\quad 00302-005+$

$00304-002+$

IMPACT SPEED VS HORIZCNTAL TRAVEL OF STRUCK EMPTY CAR

UNC

VIBRATI CN ENVIRONMENT PRGDUCEC DURING RAIL TRANSPOKT

UNC

SHCCK, RAIL HUMPING 3.4 TO $10 \mathrm{MPH}, 3$ DIRECTIONS

$00305-008+$

SHCCK RAIL HUMP SPECIAL DRAFT CAR, TO IZMPH LUNGITUDINAL

$00309-001+$

$00366-001+$

SHOCK SPECTRA, BOXCAR, LONGITUOINAL AXIS

SHOCK SPECTRA, BOXCAR, VERTICAL AXIS

SHGCK SPECTRA, BOXCAR, LATERAL AXIS

SHOCK SPECTRA, SERIES 500 ATMX CAR, LONCITUDINAL AXIS

SHCCK SPECTRA,SERIES 500 ATMX CAR, VERTICAL AXIS

SHOCK SPECTRA, 500 SERIES ATMX CAR, LATFRAL AXIS

SHOCK SPECTRA, SERIES 600 ATMX CAR, ALL AXES

SHOCK SPECTRA,HAMMER CAR, SIX-ATMX CAR TRAIN

SHCCK SPECTRA, IST STRUCK CAR, SIX-ATMX CAR TRAIN

SHOCK SPECTRA, 2ND STRUCK CAR, SIX-ATMX CAR TRAIN

SHOCK SPECTRA,3RD STRUCK CAR, SIX-ATMX CAR TRAIN

SHUCK SPECTRA, 4 TH STRUCK CAR, SIX-ATMX CAR TRAIN

SHOCK SPECTRA.5TH STRUCK CAR. SIX-ATMX CAR TRAIN

SHOCK/VIBRATION SUMMARY, UNION CARBIDE RAIL TEST

SHOCK DATA.UNION CARBIDE RAIL TEST

SWITCHING SHOCK, CUSHICN UNOERFRAME BCXCAP

01033-010+

UNC $\quad 01033-010+$

UNC 01204-057t

UNC 01205-064+

UNC $01206-059+$

UNC $01211-081+$

UNC $01212-053+$

UNC $01213-018+$

UNC $01214-035+$

UNC $01216-098+$

UNC $01217-028+$

UNC $01218-015+$

UNC $01219-085+$

UNC $01220-015+$

UNC $\quad 01221-015+$

UNC 01315-007t

UNC 01317-033+

UNC $\quad 01326-055+$

SPEEDS, RAIL SWITCHING, DISTRIEUTION

......... SHOCK............ INPUT ..... TKANSPORTATICA......... SHIP........

SHOCK SPECTRA, SHIP, TYPICAL UNC 00333-0C1+

ACCFLERATION IN CARGO SHIPS DURING MANEUVERS UNC O1133-003+

$\ldots \ldots . . .$. SHCCK........... INPUT .... TRANSPORTATION ......... OTHER .......

SUMMARY, CYNAMIC ENVIFONMENT OF TRANSPDRTATICA: - 197? UNC A1354-018+ SHOCK AND ACCELERATION DATA FCF VARIOUS MEANS TF TKANSFCET UNC SHOCK + VIERATION DATA OBTAINFL FFLM TRUCK + DAIL SHIPMEPT UNC OIOO2-OCE+

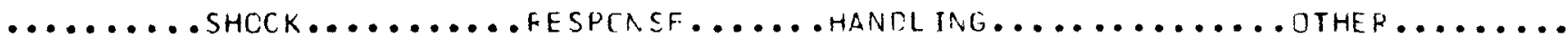

SHUCK, FORKLIFT SUMMARY REPUKT (STE 174h TO 1752)

SHUCK, VIBRATION, 2000 LB FURKLIFT TRUCK (SE 1744 )

UNC J1744-001+

UNC $31745-047+$ 


\begin{tabular}{|c|c|c|}
\hline $\begin{array}{l}\text { SHCCK, RESPONSE, } 3000 \text { LB FCFKLIFT TKUCK (SEE } 1744) \\
\text { SHCCK, RESPONSE, } 4000 \text { B FORKLIFT TRUCK (SEF } 1744) \\
\text { SHCCK, VIBEATION, } 7000 \text { LB FORKLIFT TRUCK (SEE } 1744 \text { ) }\end{array}$ & $\begin{array}{l}\text { UNC } \\
\text { UNC } \\
\text { UNC }\end{array}$ & $\begin{array}{l}21747-057+ \\
21750-058+ \\
01752-r .41+\end{array}$ \\
\hline
\end{tabular}

......... SHCCK...........FESPLASE ... TRANSPRRTATILA.......AUTOMCEILE ......

SHOCKIVIBRATICN SUMAAFY, UNICA CAFRIOF TRUCK TEST UNC O1312-0C7+ SHOCK DATA.UNICN CARBIDE TRUCK TEST

UNC DI314-013+

........ SHOCK..........RESPCVSE....TR ANSPORTATIOH.......FAILROAD......

PAIL HUMP SHOCK CRITERIA SUMMAFY

BOXCAP SHOCK CRITERIA SUMMARY

ATMX CAR SHOCK CRITER IA SLMMARY

SHOCK/VIBRATION ENVIKCNMENT SUMMARY, KAIL TRANSPORT

SHOCK SPFCTRA SUMMARY, SIX-ATNX CAK TRAIV

SHOCK SPECTRA, BOXCAR, LUNGITUDINAL AXIS

SHOCK SPECTRA,BOXCAR,VERTICAL AXIS

SHOCK SPECTRA,BOXCAR, LATERAL AXIS

SHUICK SPECTRA, SERIES SCO ATMX CAR, LCNGITUNINAL AXIS

SHCCK SPECTRA, SERIES 500 ATMX CAR, VERTICAL AXIS

SHCCK SPECTRA, 500 SERIES ATMX CAR, LATEKAL AXIS

SHCCK SPECTRA, SERIES 600 ATMX CAR, ALL AXES

SHCCK SPECTRA,HAMMER CAR, SIX-ATMX CAK TRAIN

SHOCK SPFCTRA,IST STRUCK CAR, SIX-ATMX CAR TRAIN

SHOCK SPECTRA, 2ND STRUCK CAR, SIX-ATNX CAR TRAIN

SHOCK SPECTRA,3RD STRUCK CAR, SIX-ATMX CAF TRAIN

SHCCK SPECTRA, 4TH STRUCK CAR, SIX-ATMX CAR TRAIN

SHOCK SPFCTRA, 5 TH STRUCK CAR, SIX-ATMX CAF TRAIN

SHUCK/VIHRATION SUMMARY.UNIOA CARRIOE PAIL TFSI

SHOCK DATA,UNION CARBIDE RAIL TEST

SWITCHIAG SHOCK,CUSHICN UNDFRFRAME HCXCAR

$\begin{array}{ll}\text { UNC } & \text { A1202-004t } \\ \text { UNC } & \text { B1203-002t } \\ \text { UNC } & B 1210-002+ \\ \text { UNC } & B 1320-011+ \\ \text { UNC } & 01215-012+ \\ \text { UNC } & 01204-057+ \\ \text { UNC } & 01205-064+ \\ \text { UNC } & 01206-059+ \\ \text { UNC } & 01211-081+ \\ \text { UNC } & 01212-053+ \\ \text { UNC } & 01213-018+ \\ \text { UNC } & 01214-035+ \\ \text { UNC } & 01216-098+ \\ \text { UNC } & 01217-028+ \\ \text { UNC } & 01218-015+ \\ \text { UNC } & 01219-085+ \\ \text { UNC } & 01220-015+ \\ \text { UNC } & 01221-015+ \\ \text { UNC } & 01315-007+ \\ \text { UNC } & 01317-033+ \\ \text { UNC } & 01326-055+\end{array}$

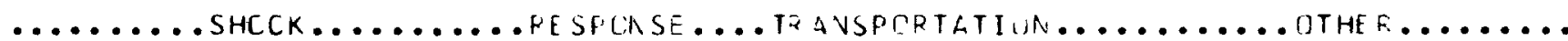

SHOCK + VIBRATION DATA CBTAINEL FFCM TKUCK + FAIL SHIPMENT IINC OICC3-ONE+

(IMPACT)

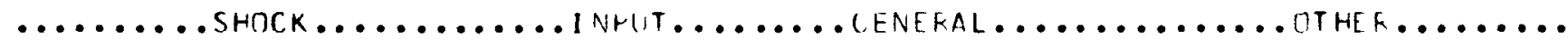

APFRCXINATE CURATICA CF SCME SHLFT ACCFLEKATITN LFIALS

UNC $\quad 00734-\mathrm{CCl}_{1}+$

( I MPACT)

$\ldots . . . .$. SHCCK............ INPUT........ANULIAG............ THEF........

NAXIMLM OROPS, PACKAGES IN THGRSIT

UNC $01609-0 \mathrm{C} 4+$ 


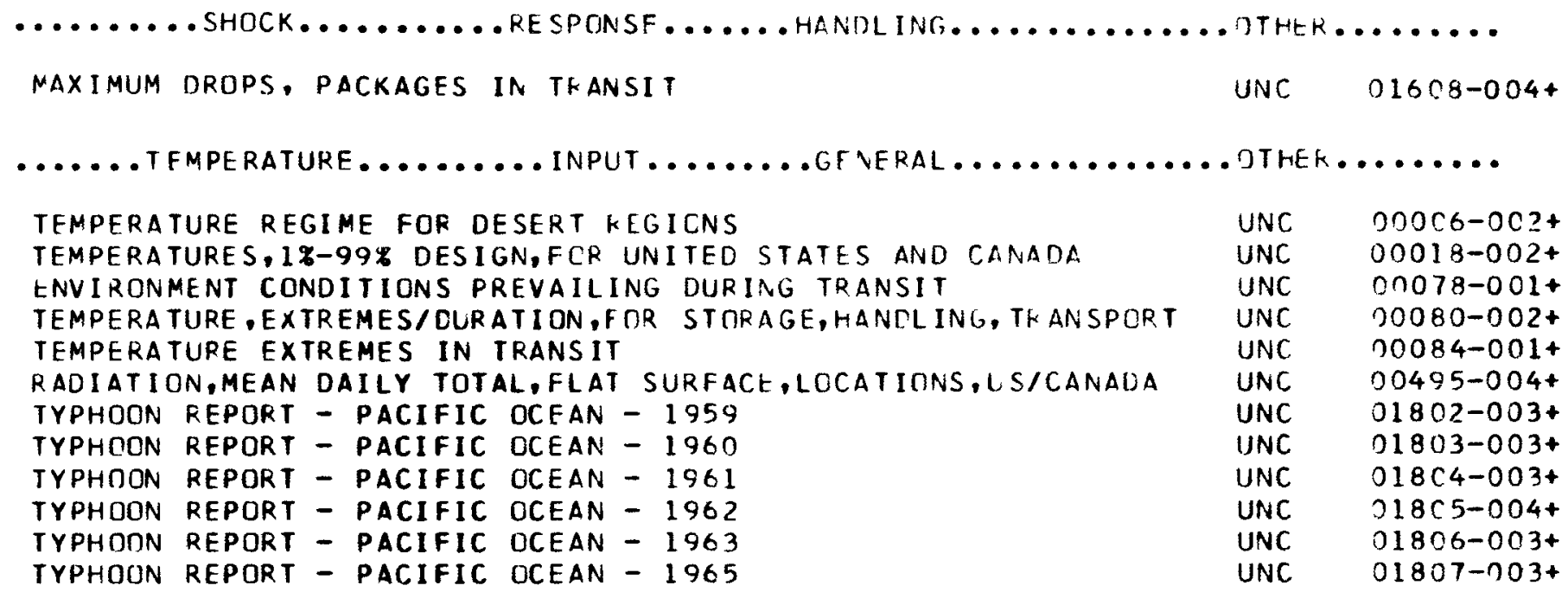

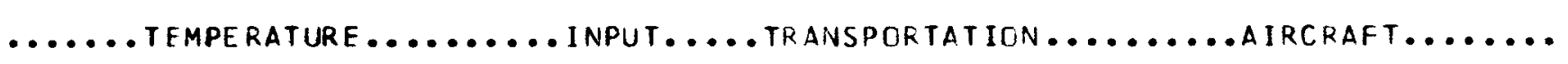

TEMPERATURES, CARGO COMPARTMENT, CII9C AIRCRAFT, ALASKA,WINTFR UNC OOOEI-COI+

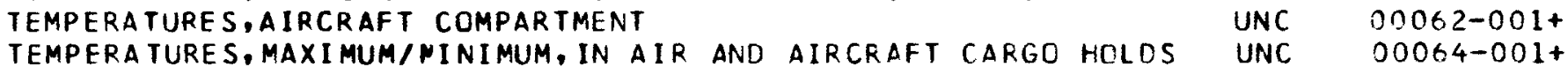
TEMPERATURES, COMPARTMENT, C141A $\quad$ UNC DO066-001+ FREQUENCY OF OCCURENCE OF AIRCRAFT ICING PARAMETERS UNC OIOR9-0C2+ IEMP/TIME DATA AIRCRAFT, TRUCK, TRAIN.ATMX UNC OI288-004+

$\ldots \ldots . .$. TEMPERATURE ......... INPUT.... TRANSPORTATION.......ALTOMOBILE.....

TEMPERATURE/TIME DATA.TRUCK (SEE 1288) UNC O13C9-004+ TFMPERATURE, TRUCK CARGO, HIGH AND LOW UNC

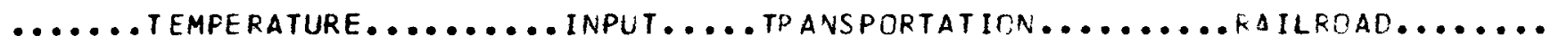

TEMPERATURE, INTERNAL AIR, RAILRLAD BOXCAR IN DESERT
TEMPERATURE TESTS. INTERNAL, RAILROAD FRFIGHT CARS WINTEF

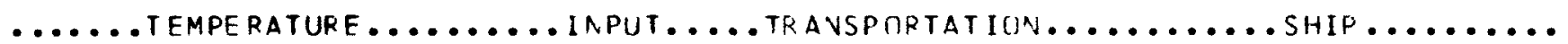

TEMPFRATURES AND RELATIVE HUMILITY,SHIP HLLU UNC ONOER-OCI+ TFMPFPATURES,MAXIMUM AND MINIMUM, AT SEA UNC UODF9-00I+ HUMIDITY, RELATIVE, ABOARD SHIP $\quad$ UNC $30232-0 C 1+$

TEMPERATURE IN SHIPS MOLDS UNC J12A2-DO3+ 


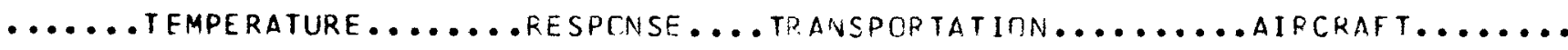

TFMP/TIME DATA AIRCRAFT, TRUCK, TRAIN, ATIMX

UNC J1288-0C4t

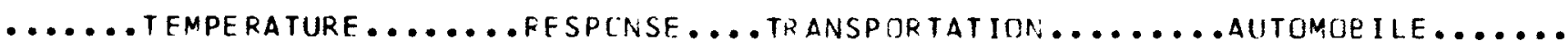

TEMPERATLRE/TIME DATA,TRUCK ISEE $12881 \quad$ UNC J13C9-OC4+ TEMPERATURE. TRUCK CARGO, HIGH AND LOW UNC O1327-027+

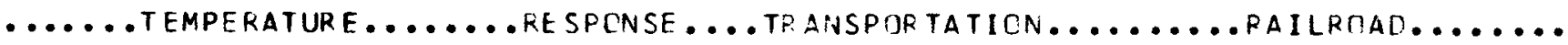

TEMPERATUFE/TIME DATA. RAIL ANL ATMX CARS ISEE 1288) UNC OI310-004+

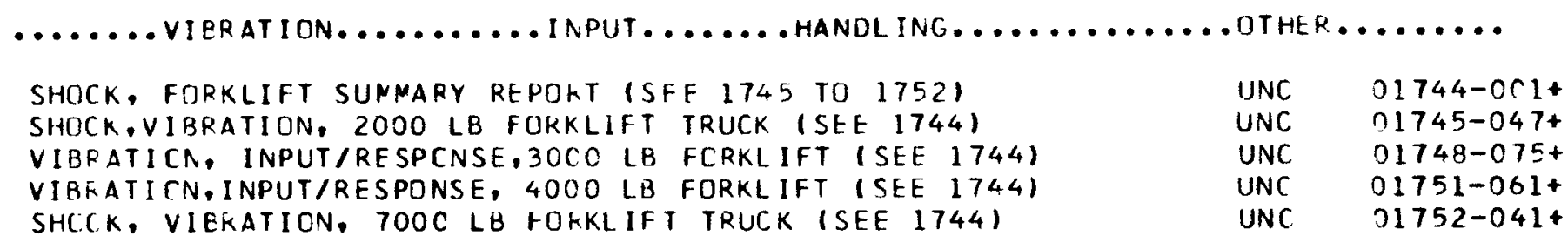




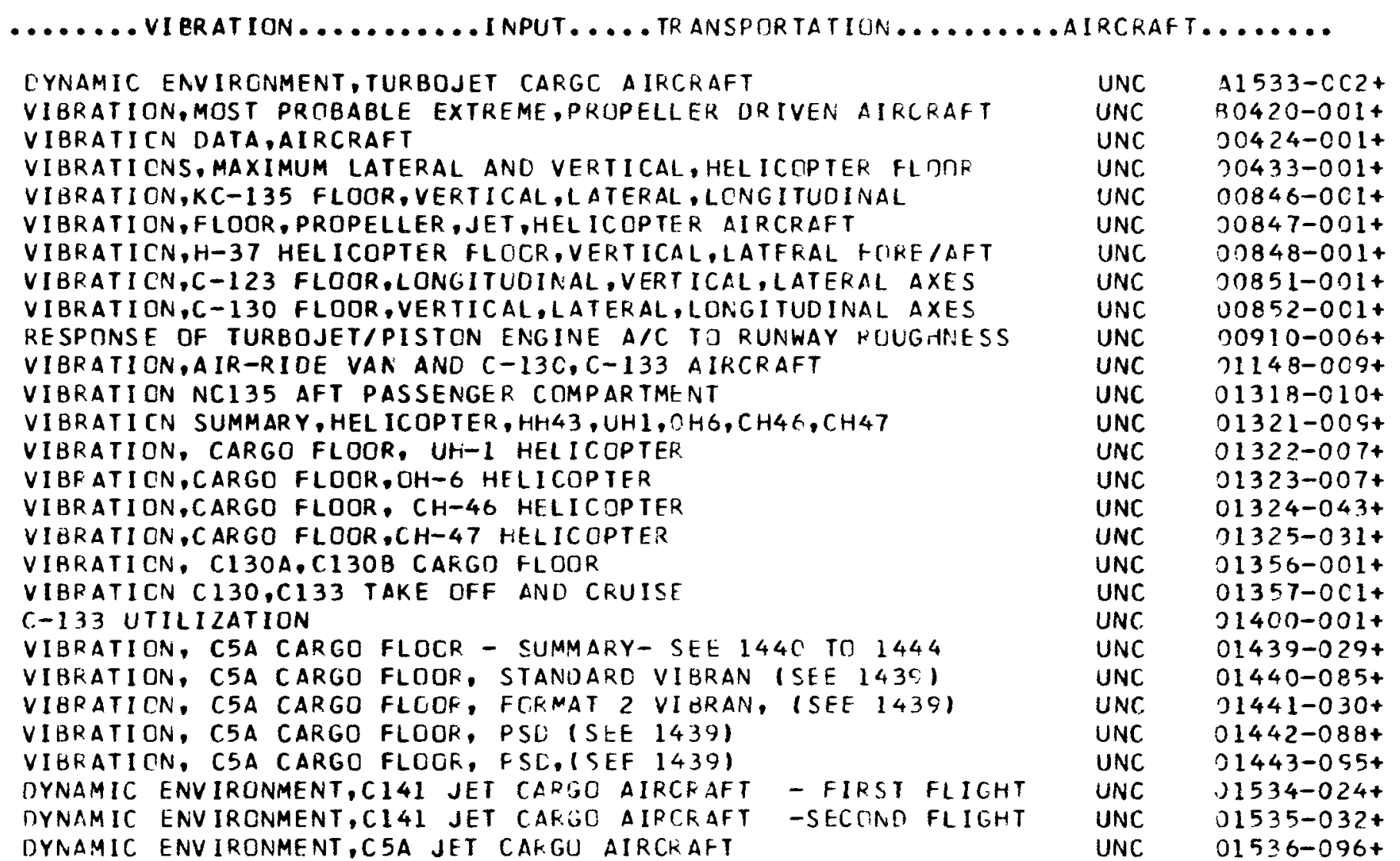

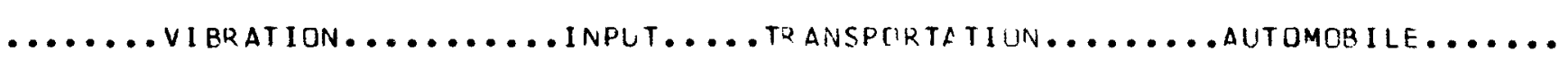

VIBKATICN-ACCELERATIOAS IN HIGHKAY OPERATICA CF TRUCKS UNC OO373-OC1+ VIBFATION IN RDAD VEHICLES UNC OO374-001+ VIRRATICN,MAXIMUM, CN TRUCKBED,VARIETY EF FLIAD CPFRATICAS OOST7-DC1+ VIBRATION.MAXIMUM ACCFLERATICA IN CARGD TKUCK UNC VIEKATICN DATA, TYPICAL, VAN TFAILER, NCRMAL SUSPENSION UNC OO397-OCI+ VIBKATION,FLAT-BFD TRACTOR-TKAILFF VIBFATICN.TRUCK FLOOR.EMPTY ANU FULLYLCADEC UN849-0CI+ 


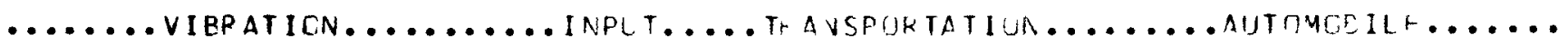

DYNANIC ENVIRCNMENT OA A FLAT BED TRACTOR/TRAILEF IAHC/CICL

UNC

FINAL ANALYSIS,AFCIDOD FLATEED TRUCK TEST

VIBRATICN ENVIRONMENT, AEC VAN, WITH TRANSIT RIG SHIHPING CONT

VIBKATICN/SHOCK, $21 / 2$ TON TRUCK

VIBRATICA/SHCCK AIR SLSPENSICA VAN

SHOCK/VIBRATION SUMMARY, UNITA CAR EIDF TRUCK TFST

VIBRATICN DATA, UNION CAKEIDE TRUCK TFST

VIBFATION, TRUCK, TANDEM AXLE, AIK SAU SUSPENSITN

VIBRATICN IA COMMCN MCTOR CAFRIERS
UNC

UNC

UNC

UNC

UNC

UNC

UNC

UNC
$21006-008+$ j1083-041t $1245-061+$ $012+4-002+$ $01277-006+$ $01312-007+$ $01313-045+$ $01361-001+$ 01742-007t

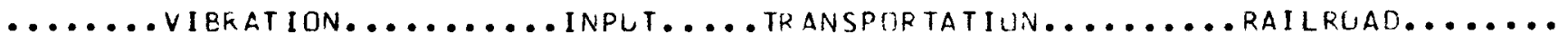

SHICK/VIERATION ENVIRCNMENT SUMMARY, FAIL TRANSPTFT

VIBRATION,RAIL TRANSPCRT, SUMMARY UF DATA UN

VIBPATICN DATA,RAIL

VIBFATION TESTS, RAILROAD, SPEED $4 O M P H$

VIBEATICN TESTS,RAILRCAC, SPEER $73 \mathrm{MPH}$

VIGFATICN TESTS, RAILRCAO,SPEEU $80 M$ PH

VIEKATINNS IN RAILROAC FREIGHT CARS AT SPFEDS FPEM $21-73 M P H$

VIBRATICNS MEASURED ON ATMX 5OC CAR AT SPET DS 30-7OMPH

VIBKATIUN EAVIRONMENT PEODUCEC DURING KAIL TRANSPCRT

VIBRATICN,RAILROAD, SOFT SPRUNG CAR, VERTICAL ANC LATEFAL

VIBFATICN, RAILRCAD CCNTINUUUS AND TRANSIFNT

VIBPATICN, RAILROAD ENVELLPES CIKECTICNAL COMPRSITIS

SHCCK/VIBPATION SUMMARY, UNIUN CARHIDE KAIL TEST

VIBEATION DATA.UNION CARBIDE RAIL TEST

UNC B1320-011t

UNC $30355-001+$

UNC $00357-001+$

UNC $\quad 0358-001+$

UNC 00359-001+

UNC $\quad 003+0-001+$

UNC $00362-001+$

UNC ग0363-001+

UNC $00366-001+$

UNC 00850-001+

UNC 31031-0C1+

UNC $\quad 01032-001+$

UNC $\quad 91315-007+$

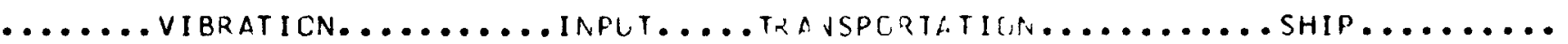

VIEKATICN,MOST EXTREMF PRCBABLF,FXPECTEL IN SFA TRANSPCIFT HOAO4-0OL MEAT EXTREME PRC BABLE OFXPECTEL VIBRATICN DATA, SHIPS

VIBKATION DATA.SHIPS IN STRAIGHT FUNS AT VARIOUS SPFFIS

VIBKATICN, SHIPBOARD,NCRMAL STEGMING ANI HAPD TUFNS

VIBKATIGN DATA,SHIPS, HIGH SPEED MANFUVHR ANT CFASI, EACK

VIBFATICNS, SHIPBOARD, FROM VARILUS SOUPCES

VIEKATIONS, MERCHANT SHIP SS WULVHKINE STATF

$\begin{array}{ll}\text { UNC } & 30202-001+ \\ \text { UNC } & 00405-001+\end{array}$

UNC $\quad 20407-001+$

UNC $\quad 30408-001+$

UNC $\quad 30409-001+$

UNC J3415-001+

UNC $00418-025+$

.......VIBRATION.......... IP.PUT.... TKLVSPTFTATITN.........กTRER........

SUMPARY, OYNAMIC ENVIRUAMFNT CF TRANCP IF TATICA - 1972

VIBRATION-MAXIMUM ACCELEKATITAS FRK VATIOUS VEHICLES

SHCCK + VIBEATION OATA OBTAIMELFRLM THUCK + FAIL SH IPMENT

TFANSPORTATION VIBRATION FNVIRIAMEITS

NNC $41354-018+$

JNC $00368-001+$

UNC $01003-006+$

UNC $01176-002+$ 


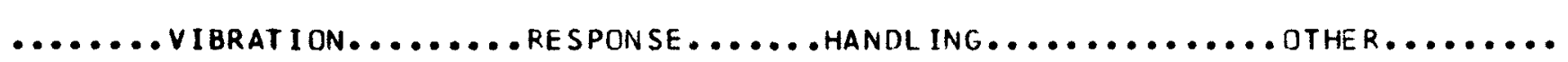

SHOCK, FORKLIFT SUMMARY REPORT (SEE 1745 TO 1752 )

SHOCK,VIBRATION, 2000 LB FORKLIFT TRUCK (SEE 1744)

UNC

$01744-0 \mathrm{Cl}+$

VIBRATICN, INPUT /RESPCNSE, 3000 LB FORKL IFT (SEE 1744)

VIBRATION, INPUT/RESPONSE, 4000 LB FORKLIFT (SEE 1744)

UNC

$01745-047+$

SHOCK, VIBRATION, 7000 LB FORKLIFT TRUCK (SEE 1744)

UNC

$01748-075$

UNC

$01751-061+$

UNC

$01752-041+$

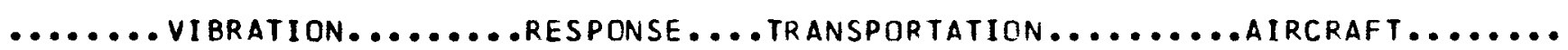

VIBRATION NCI35 AFT PASSENGER COMPARTMENT

UNC $\quad 01318-010+$

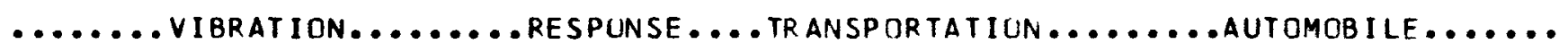

VIBRATICN ENVIRONMENT ,AEC VAN WITH TRANSIT RIG SHIPPING CONT UNC O1245-06I* SHOCKIVIBRATION SUMMARY.UNION CARBIDE TRUCK TEST

VIBFATICN DATA, UNION CARBIDE TRUCK TEST

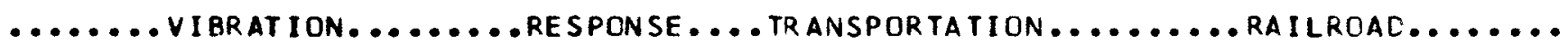

SHOCK IVIBRATION ENVIRCNMENT SUMMARY, RAIL TRANSPORT UNC B1320-011+ SHOCKIVIBRATION SUMMARY, UNION CARBIDE RAIL TEST UNC O1315-0O7+ VIBRATICN DATA,UNION CARBIDE RAIL TEST UNC O1316-065+

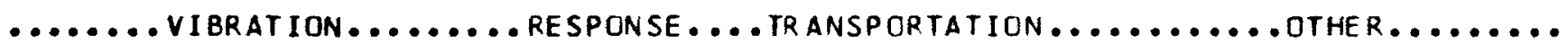

SHOCK + VIBRATION DATA OBTAINED FRCM TRUCK + FAIL SHIPMFNT UNC O1CO3-006+ 
$\ldots \ldots \ldots \ldots .$. .............. INHUT.......GENERAL..........THEF......

ENVIRONMEAT CONDITIONS PREVAILING DURING TRANSIT

WIND VELOCITIES, WORLDWIDE, AND OISTRIBUTIIJN IN TEMPFFATE 7 LNE

WIND SPEED DISTRIBUTICN IN THE UNITEC STATES

GLAZE ICE, WIND LCADS AT EARTHS SURFACE

IINC

UNC

UNC

UNC

$00258-001+$

$01595-005+$

$01722-001+$

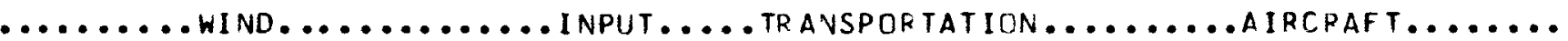

FAT IGUE DAMAGE PARAMETERS, TRANSPORT AIRCRAFT

UNC $\quad 01085-001+$

AIRCRAFT RECORDINGS DURING A TUFBULENCE INCIDENT

UNC

$01090-001+$

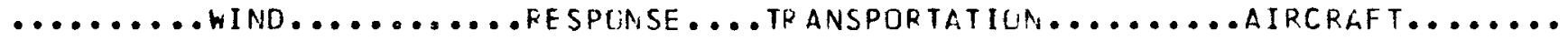

GUST LOAC FREOUENCY. AIRCFAFT FLIGHTS.40-45KILOFEET, IN STURM

AIRCRAFT RECORDINGS DURING A TURBULENCF INCIDFNT

UNC $\quad 21087-002+$

UNC $\quad 01090-001+$

\begin{tabular}{|c|c|c|c|c|}
\hline WIND ANI & Avt & HEIGHTS - & IROP I CAL & STORMS \\
\hline TYPHOON & KEPORT & - PACIFIC & CCEAN - & 1959 \\
\hline TYPHOON & REPORT & - PACIFIC & CCEAN - & 1960 \\
\hline TYPHOON & REPCRT & - PACIFIC & UCEAN - & 1961 \\
\hline TYPHONN & REPORT & - PACIFIC & OCEAN - & 1562 \\
\hline TYPHDON & REPORT & - PACIFIC & LCEAN - & 1963 \\
\hline TYPHOON & REPORT & - PACIFIC & LCEAA. - & 1965 \\
\hline TYPHOUN & REPORT & - PACIFIC & OCEAN - & 1966 \\
\hline TYPHOUN & REPORT & - PACIFIC & CCEAN - & 1967 \\
\hline TYPHOGN & REPORT & - PACIFIC & LCEAN - & 1968 \\
\hline TYPF OON & REPORT & - PACIFIC & CCEAN - & $19+9$ \\
\hline TYPHOON & REPORT & - PACIFIC & CCEAM - & 1970 \\
\hline TYFHOON & REPORT & - PACIFIC & LCEAN - & 1974 \\
\hline
\end{tabular}

GENEKAL

OTHER .........

UNC $\quad 01636-002+$

UNC $\quad 01802-003+$

UNC 91803-003t

UNC $01804-003+$

UNC $01805-004+$

UNC 01806-003t

UNC $\quad 21807-003+$

UNC $\quad 01808-003+$

UNC 01809-003+

UNC $01810-004+$

UNC $\quad 01811-002+$

UNC 01812-003+

TYPHOCN REPORT - PACIFIC LCEAN - 1974

$01813-003+$ 
Abnormal Environments 
(FARTHOUAKE)

(IMPACT)

(IMPACT)

(IMPACT)

(IMFACT)

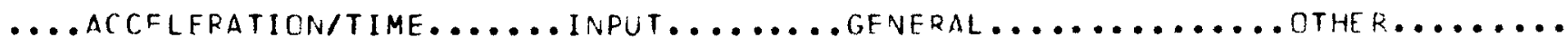

EALTHQUAKES IN THF UNITED STATHC

FARTHOUAKE. STRUNG MUTION - ACCELDGRAMS G VS IIME

EARTHGLAKE, STRONG MOTION - ACCELOGRAMS G VS TINF

EARTHGUAKE - ROCK MOTION ACCELLGRAMS G VS TIME, CISTANCE

STR NNG MOTION EARTHOUAKE ACCELOGKAMS - PAFT F

STRCNG MOTION EARTHOUAKE ACCELUGRAMS - PAFT C

SIRCNG MOTION EARTHOUAKE ACCELOGRAMS - PAFT

STRTNG MOTICN EARTHQUAKE ACCELCGRAMS- VOL I,PT I, LATA

STRCNG NOTION EARTHOUAKE ACCELUGRAMS- VCL I,PT J, CATA

STRCNG MOTION EARTHQUAKE ACCFLOGRAMS- VGL I,PT K, DATA

STRONG MOTION EARTHOUAKE ACCELOCRAMS-VOL III,RES. SFECTRA

STRCNG MOTION EARTHOUAKE ACCELOGRAMS-V'JL IV, FCURIEK SPECTRA

EARTHOUAKE, ANCHORAGF, $19 E 41$ ENGINEEPING INFORMATICA

$\begin{array}{ll}\text { UNC } & 30948-004 t \\ \text { UNC } & 31526-004+ \\ \text { UNC } & 31527-004 t \\ \text { UNC } & 01528-001+ \\ \text { UNC } & 01570-004+ \\ \text { UNC } & 01571-004 t \\ \text { UNC } & 01572-004 t \\ \text { UNC } & 01600-004+ \\ \text { UNC } & 01601-004 t \\ \text { UNC } & 01602-004+ \\ \text { UNC } & 01603-003+ \\ \text { UNC } & 01604-002+ \\ \text { UNC } & 01641-002+\end{array}$

....AC.CELERATICN/TIME.

. INPUT.

.GENFFAL

DTHER.........

APPROXIMATE DURATICA CF SCME SHLRT ACCELEKATICN LOACS

UNC

$00734-001+$

..... ACCEleration

ANALYSIS CF DIRECTICN CIF LOADS IN 1 IRCRAFT ACCIDENTS ACCELERATION OF FLOOR-AIRCRAFT CRASHES

ACCFIERATIUN, AIRCRAFT CRASH-VAKINUS ANGLES CF IMPACT

FLOCIR ACCELERATIONS. TFANSPOFT AIRCRAFT ACCIDENTS

CIVIL AVIATICN ACCIDENT DATA - 1962 T] 1972

AIRCF $\triangle F T$ ACCIDENT - ECEINC $7 C 7-8 / 28 / 73$

AIRCRAF T ACCIDENT - BCEING $747-4 / 12 / 72$

UNC $\quad 30751-0 \mathrm{C} 1+$

UNC $\quad 00752-001+$

UNC $00753-008+$

UNC $01629-001+$

UNC $\quad 01664-0 C 5+$

UNC $\quad 01801-001+$

UNC J1818-001+

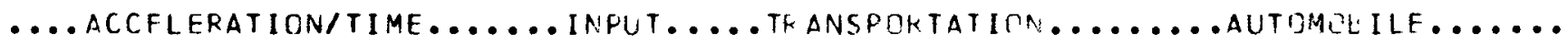

ACCELERATIONS, AUTOMOBILE CKASH (HEAC-CN) UNC $30737-0 C 1+$ DFCELERATION PATTERNS FOR AUTOMTBILE HFAD-CN (OLLISII,NS UNC 00738-001+

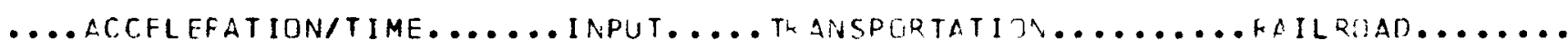

RAIL ACCICENT STATISTICS

UNC $\quad 21020-025+$ 
(PUNCT URE)

(PUNCTURE)

( PUNCTURE)

( PUNC TURE)

(PUNCTURE)

( P UNCTURF)

(PUNC TURE)

(EXPLCSION)

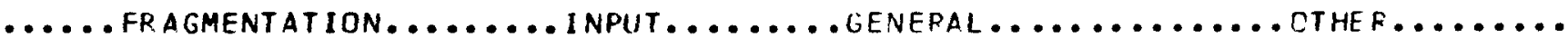

FRAGMENTS, MASS, VELOCITY, STANDARC ARTILLERY SHELLS UNC O1814-CC2+

.....FRAGMENTATION........ INPUT.... TF ANSPORTATION........AIRCRAFT......

ROTOR FRAGMENT CONTAIAMENT-JET FNGINE FAILURE - $1972 \quad$ UNC O1781-01C+

.....FRAGMENTATION........ INPUT .... TP.ANSPCRTATIGN.......AUTUMOBILE......

MOTOR CARRIER ACCIDENT ENVIRONMENT - TAC STUCY

TRUCK ACCIDENT -FLORIDA-8/8/71

TRUCK ACCIDENT REPORTS, NHTSA, NO. 7

TRUCK ACC IDENT REPORTS, NHTSA, NO. B

TRUCK ACCIDENT REPORTS, NHTSA, NI. 9

$\begin{array}{ll}\text { UNC } & 41662-007+ \\ \text { UNC } & 91695-003+ \\ \text { UNC } & 01753-005+ \\ \text { UNC } & 01754-004+ \\ \text { UNC } & 01755-0 C 5+\end{array}$

$\ldots \ldots .$. FRAGMENTATION....... INPUT .... TRANSPORTATION.......RAILROAD......

RAILROAC ACCIDENT ENVIRONMENT - TAC STUDY UNC AI663-0CE+

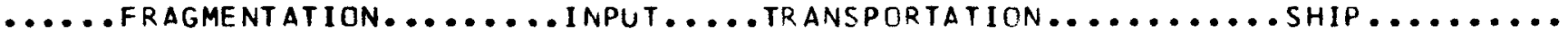

IMPACT, PENETRATION, SHIP COLLISICA ANO GROUNOING UNC OI550-001+

..... FRAGMENTATION...... RESPCNSE ... TRANSPORTATICN.......AUTOMOEILE......

CARGO RESPONSE TRUCK AND RAIL ACCIDENTS UNC O1688-0C2+

TRUCK ACCIDENT-FLDRIDA-8/8/71

UNC $01695-003+$

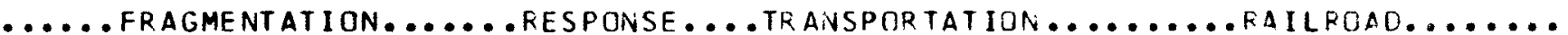

CARGO RESPONSE TRUCK AND RAIL ACCCIDENTS SEE EDB1688 UNC O1699-002+

.....FRAGMENTATION........ INPUT.... TRANSPOKTATION.......AUTOMCEILE......

TRUCK $\triangle$ ACCIDENT-NEW JERSEY $9 / 21 / 72$

TRUCK ACCIDENT-GEORGIA-6/4/71

TRUCK ACCIDENT-VIRGINIA-3/9/72 
(CRUSH)

(CRUSH)

(IMMERSION)

(EXPLCSION)

(EXPLISINN)

\begin{tabular}{|c|c|c|c|c|c|c|}
\hline TYPHOCN & REPURT & - PACIFIC & CCEAN. & -1959 & UNC & $01802-003+$ \\
\hline TYPHOON & REPORT & - PACIFIC & CCEAN & -1960 & UNC & $01803-003+$ \\
\hline TYPHOCN & REPORT & - PACIFIC & OCEAN & -1961 & UNC & $01804-003+$ \\
\hline TYPHOON & REPORT & - PACIFIC & DCEAN & -1962 & UNC & $01805-004+$ \\
\hline TYPHCON & REPCRT & - PACIFIC & CCEAN: & -1563 & UNC & $01806-003+$ \\
\hline TYPHOON & REPORT & - PACIFIC & OCtAN & -1965 & UNC & $01807-003+$ \\
\hline TYPHDON & REPORT & - PACIFIC & OCEAN & -1966 & UNC & $01808-003+$ \\
\hline TYPHIOON & REPORT & - PACIFIC & CCEAN & -1967 & UNC & $01809-003+$ \\
\hline TYPHOON & REPORT & - PACIFIC & CCEAN & -1968 & UNC & $01810-004 t$ \\
\hline TYPHOON & REPORT & - PACIFIC & CCEAN & -1969 & UNC & $01811-002+$ \\
\hline TYPHOON & REPORT & - PACIFIC & CCEAN & -1970 & UNC & $01812-0034$ \\
\hline TYPHOON & REPORT & - PACIFIC & CCEAN & -1974 & UNC & $01813-003+$ \\
\hline
\end{tabular}

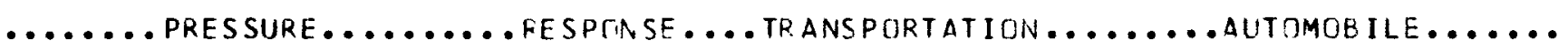

CARGO RESPONSE TRUCK AND RAIL ACCICENTS

UNC $\quad 01688-002+$

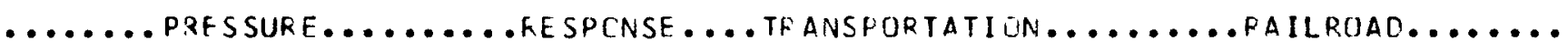

CARGR RESPONSE TRUCK AND KAIL ACCCICENTS SEE EGBIGE8 UNC O1699-002+

....... PRESSURE .......... INPUT .... TRANSPRFTATIUN........AIRCRAFT ......

A/C ACCICENT DEHAVILLAND CHC 16 FEB $1970 \quad$ UNC O1679-0C3+

A/C ACCIDENT DC9 MAY $1970 \quad$ UNC $1682-003+$

A/C ACCIDENT SKYVAN SERIES 3 JULY $1970 \quad$ UNC O1683-0C3+

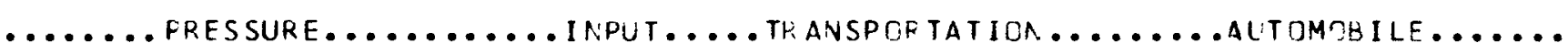

HYCRDGEN TRANSPORT ANC HANCLINC EXPLCSILV UNC O1758-OC1+

.......PRESSURE.......... INPUT .... TPANSPORTATICN.......RAILRUAC......

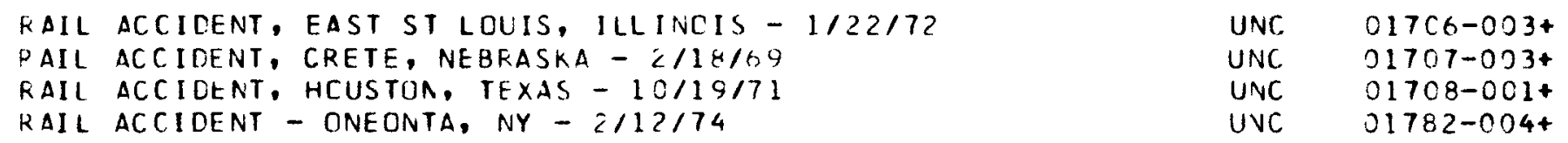


( LIGHTNING)

(LIGHTNING)

ICRUSHI

(IMPACT)

(IMPACT)

(CRUSH)

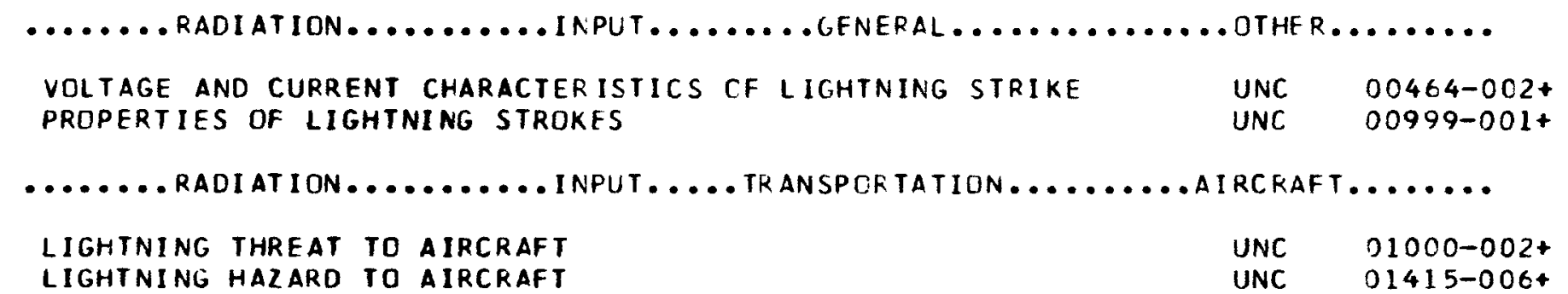

$\ldots \ldots . . . . .$. SHOCK

MOTOR CARRIER ACCICENT ENVIRCNMENT - TAC STUCY

TRUCK ACCIDENT REPORTS, NHTSA, NO. 7

TRUCK ACCIDENT REPORTS, NHTSA, NO. 8

TRUCK ACCIDENT REPCRTS, NHTSA, NT. 9 $\begin{array}{ll}\text { UNC } & \text { A1662-007t } \\ \text { UNC } & 01753-005+ \\ \text { UNC } & 01754-004+ \\ \text { UNC } & 01755-005+\end{array}$

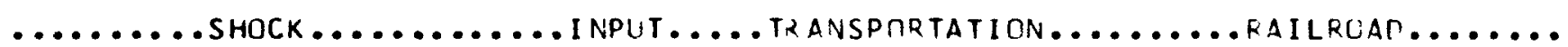

RAILROAD ACCIDENT ENVIRONMENT - TAC STUOY

UNC

A1663-006t

......... SHOCK............ INPUT

- GENERAL

APPROXIMATE DURATICN CF SCME SHORT ACCELERATICN LOADS

UNC

$00734-001+$

$\ldots . . . .$. SHOCK........... INPUT

HANDL ING.

OTHER........

IMPACTS CUE TO LARGE CRCPS WHEN LTACING;

MAXIMUM DKOPS, PACKAGES IN TKANSIT
UNC $\quad 00342-0 \mathrm{Cl}+$ 


\begin{tabular}{|c|c|c|}
\hline AIRCRAFT ACCIDENT ENVIFONMENT - TAC STUDY & UNC & $41630-004+$ \\
\hline ANALYSIS OF DIRECTION CF LOADS IN AIRCRAFT ACCIDENTS & UNC & $30751-001+$ \\
\hline ACCELERATION OF FLOOR-AIRCRAFT CRASHES & UNC & $00752-001+$ \\
\hline ACCFLERATION, AIRCRAFT CRASH-VAFIOUS ANGLES OF IMPACT & UNC & $00753-008+$ \\
\hline CRASH TFST,DC-7 AIRCRAFT & UNC & $01104-007+$ \\
\hline CRASH TEST, CONSTELLATION AIRCRAFT & UNC & $01105-018+$ \\
\hline FLOCR ACCELERATIONS, TRANSPIBT AIRCRAFT ACCICENTS & UNC & $21629-001+$ \\
\hline CIVIL AVIATION ACCIDENT DATA - 1962 TO 1972 & UNC & $01664-005+$ \\
\hline ANALYSIS OF CARGO A/C CRASH RECORUS CAB OATA & UNC & $01668-005+$ \\
\hline CRASH IMPACT CONDITICNS, MULTI HNGINE TRANSFCFT A/C & UNC & $01670-029+$ \\
\hline SURVEY CF A/C CRASH SURVIVABILITY, CARGO AT A/C CG & UNC & $01671-004 t$ \\
\hline ACCIDENT DATA, MULTIENGINE AIRCRAFT & UNC & $01672-010+$ \\
\hline A/C ACC IDENT DC 8 E2 SEPT 1970 & UNC & $01676-003+$ \\
\hline A/C $\triangle C C I D E N T$ MARTIN 404 OCT 1970 & UNC & $01677-003+$ \\
\hline A/C ACC IDFNT CONVAIR 580 DEC 1968 & UNC & $01678-003+$ \\
\hline A/C ACCIDENT UEHAVILLAND CHC IE FEB $197 \mathrm{C}$ & UNC & $31679-003+$ \\
\hline A/C ACCIDENT DOUGLAS C54D APRIL 1970 & UNC & $01680-003+$ \\
\hline$A / C$ ACC IDENT DC 9 JAN 1970 & UNC & $01681-003+$ \\
\hline A/C ACCIDENT DC9 MAY 1970 & UNC & $01682-003+$ \\
\hline A/C ACCIDENT SKYVAN SERIES 3 JULY 1970 & UNC & $01683-003+$ \\
\hline$\triangle / C$ ACCIDENT DC9 32 SEPT 1970 & UNC & $01684-003+$ \\
\hline A/C ACCIDENT DC 8 G3F SEPT 1970 & UNC & $01686-003+$ \\
\hline AIRCRAFT ACCIDENT REPCRTS - NTSB - 1972 & UNC & $01724-002+$ \\
\hline AIRCRAFT ACCIDENTS, TYPE, RATE, NTSB 1970 & UNC & $01756-002+$ \\
\hline AIRCPAFT ACCIDENTS, TYPE, RATE, NTSB 1973 & UNS: & $01757-001+$ \\
\hline AIRCRAFT $\triangle C C I D E N T$ - BCE ING $707-1 / 16 / 74$ & UNC & $21800-001+$ \\
\hline
\end{tabular}

(IMPACT) ........ SHOCK........... INPUT.... TI AINSPCPTATION........AIRCFAFT ......

AIRCRAFT CRASH ENVIROAMENTS - PIAVY

AIRCRAFT ACCIDENT - CCNVAIR EOC - $9 / 27 / 73$

AIRCRAFT MICAIR COLLISIONS

AIRCRAFT DITCHING INVESTIGATION

UNC

UNC

UNC

UNC
$01819-001+$ $01820-001+$ $01822-002+$ $01823-002+$ 
(I MPACT)

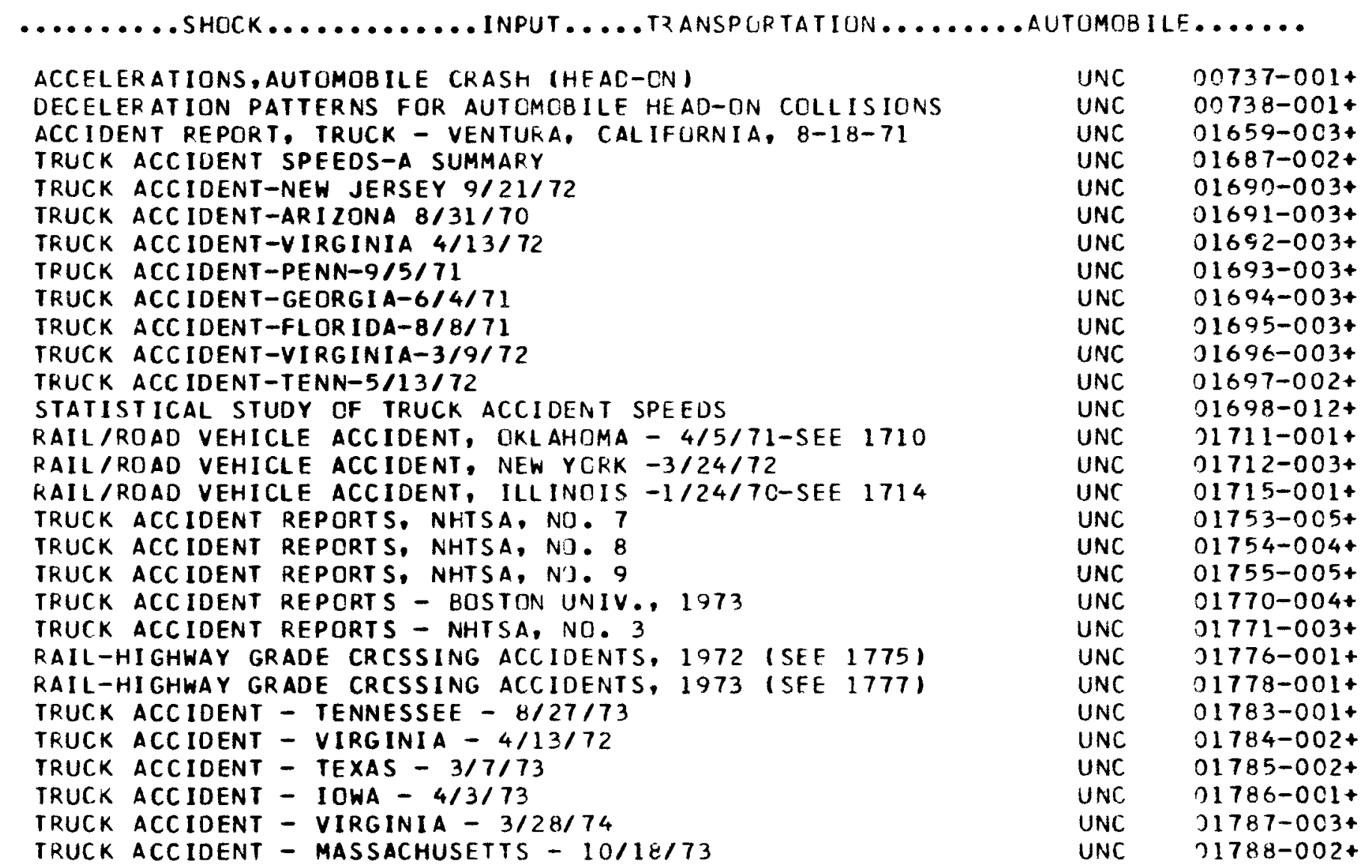

(IMPACT)

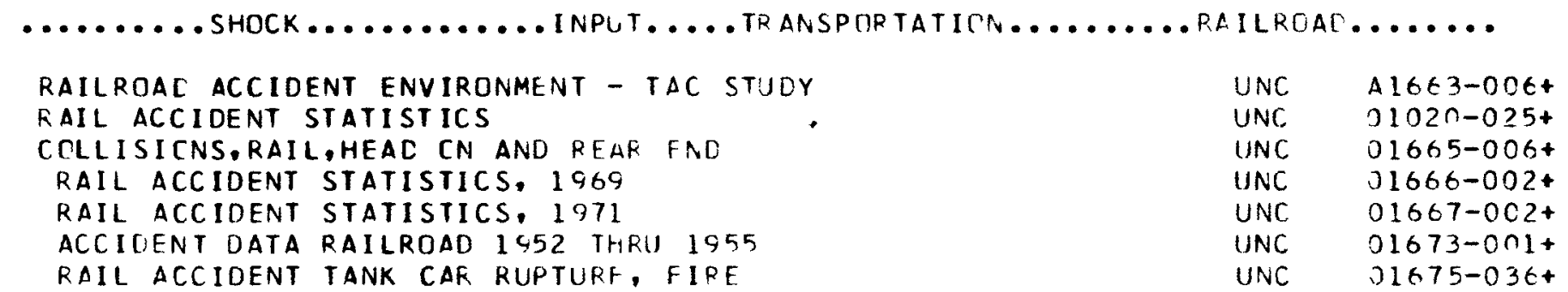


(IMPACT) SHOCK INPUT

KAILRLAC.......

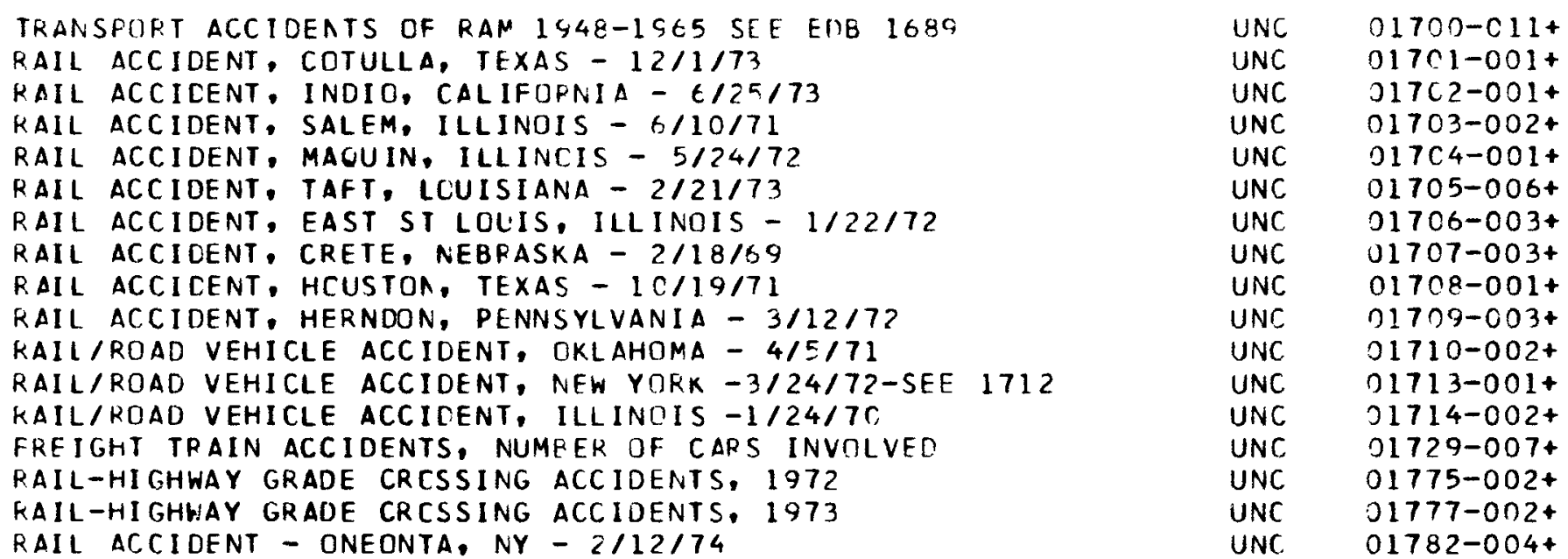

(I MPACT)

( I MPACT)

(IMPACT)

( I MPACT )

(IMPACT)
$01777-002+$
$01782-004+$

IMPACT, PENETRATION, SHIP COLLISICN ANU GHQUNTING UNC OLFO-OCI+ COLLISICN - TUG/BARGE WITH BRIOGE

$\ldots \ldots \ldots \ldots . .$. SHOCK......... FESPUASE ..... HANDL ING ..........OTHER ......

MAXIMUM DROPS, PACKAGES IN TKANSIT UNC J1608-004+

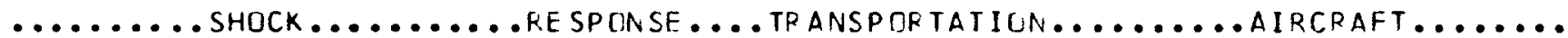

SHCCK SPECTRA.STAGED A/C CRASH TESTS UNC O1669-018+

SURVEY CF A/C CRASH SURVIVABILITY, CAFGO AT A/C CG UNC

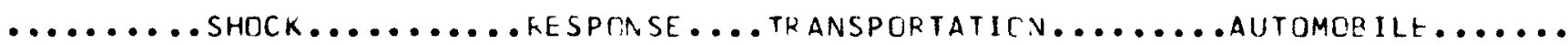

CARGO RESPONSE TRUCK AND RAIL ACCIDFNTS

UNC $\quad 01688-002+$

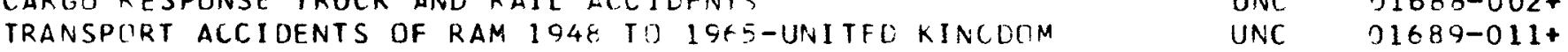

TKUCK ACCIDENT-FLORIDA-8/8/71 UNC O1695-003+

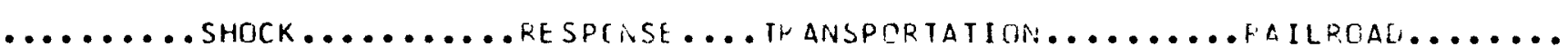

CARGO RFSFONSE TRUCK AND RAIL ACCIDENTS SEE Fl991688 UNC DI699-002+ 


\begin{tabular}{|c|c|c|c|c|c|}
\hline TYPHOON & REPORT & - PACIFIC & CCEAN - 1959 & UNC & $01802-003+$ \\
\hline TYPHOCN & REPORT & - PACIFIC & CCEAN - 1960 & UNC & $01303-003+$ \\
\hline TYPHOON & REPORT & - PACIFIC & CCEAN - $19 \in 1$ & UNC & $01804-003+$ \\
\hline TYPHOON & REPCRT & - PACIFIC & OCEAN - 1962 & UNC & $01805-004+$ \\
\hline TYPHOON & REPORT & - PACIFIC & DCEAN - $196 ?$ & UNC & $01806-003+$ \\
\hline TYPHOON & REPORT & - PACIFIC & DCEAN - 1965 & UNC & $01807-0038$ \\
\hline
\end{tabular}

(FIRF)

DN REPORT - PACIFIC OCEAN - 196

. GENERAL.............. OTHEF ........

FIRES,L IOUID HYDROCARBON FUEL,HEAT TRANSFER IN UNC OO517-011+ BURNING CONDITIONS OF PETROLEUM LIQUIDS UNC $0518-001+$

(FIRE)

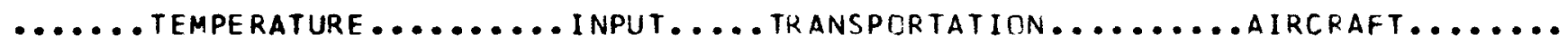

AIRCRAFT ACCIDENT ENVIRONMENT - TAC STUDY

CIVIL AVIATION ACCIDENT DATA - 1962 TO 1972

FIRE INFCRMATION AIRCRAFT ACCIDENTS

$A / C$ ACC IDENT MARTIN 404 OCT 1970

A/C ACCIDENT CONVAIR 580 DEC 1968

A/C ACCIDENT DOUGLAS C54D APRIL 1970

$\triangle / C$ ACCIDENT 74712 SEPT 1970

A/C ACCIDENT DC8 63F SEPT 1970

AIRCRAFT ACCIDENT REPCRTS - NTSB - 1972

AIRCRAFT ACCIDENTS, TYPE, RATE, NTSB 1970

AIRCRAFT ACCIDENTS, TYPE, RATE, NTSB 1973

AIRCRAFT ACCIDENT - BCEING 707 - $1 / 16 / 74$

AIRCRAFT ACCIDENT - DC $B-6 / 20 / 73$

$\begin{array}{ll}\text { UNC } & \text { A } 1630-004 t \\ \text { UNC } & 01664-009 t \\ \text { UNC } & 01674-002+ \\ \text { UNC } & 01677-003+ \\ \text { UNC } & 01678-003+ \\ \text { UNC } & 01680-003+ \\ \text { UNC } & 01685-003 t \\ \text { UNC } & 01686-003+ \\ \text { UNC } & 01724-002+ \\ \text { UNC } & 01756-002+ \\ \text { UNC } & 01757-001+ \\ \text { UNC } & 01800-001+ \\ \text { UNC } & 01821-001+\end{array}$

(FIRE)

(f IRF)

...... TEMPERATURE ......... INPUT..... TH ANSPCRTATION.......AUTDMOB ILE......

MOTOR CARRIER ACCIDENT ENVIRCNMENT - TAC STUCY

UNC A16t2-007t FIRE TEMPERATURES-TRUCK

AC.CIDENT REPORT. TRUCK - VENTURA, CALIFORNIA, 8-18-7I

TRUCK ACCIDENT-VIRGINIA $4 / 13 / 72$

TRUCK ACCIDENT-PENA-9/5/71

TRUCK ACCIDENT-TENN-5/13/72

TRUCK ACCIDENT REPCRTS. NHTSA, NJ. 8

HYDPOGEN TRANSPORT AND HANULING FXPLOSICIN

TRUCK ACCIDENT - VIRGINIA - 4/13/72

TRUCK ACCIDENT - TEXAS - 3/7/73

TRUCK ACCIDENT - MASSACHUSETTS - 10/18/73

$\begin{array}{ll}\text { UNC } & 00520-001+ \\ \text { UNC } & 01659-003+ \\ \text { UNC } & 01692-003+ \\ \text { UNC } & 01693-003+ \\ \text { UNC } & 01697-002+ \\ \text { UNC } & 01754-004+ \\ \text { UNC } & 01758-001+ \\ \text { UNC } & 01784-002+ \\ \text { UNC } & 31785-002+ \\ \text { UNC } & 01788-C C 2+\end{array}$

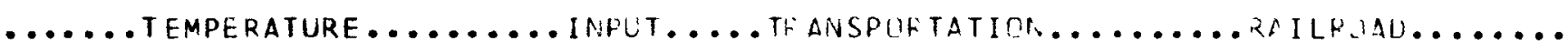

RAILKUAC ACCIDENT EAVIRONMENT - TAC STHITY

FIRF TFMPERATURES-RAIL

UNC A $1663-006+$
IVNC 
(FIRE)

(FIKE)

(F IRF)

(STCRM)
ACCIDENT DATA RAILROAD 1952 THRU 1955

RAIL ACCIDENT TANK CAR RUPTURE. FIRE

TRANSPORT ACCIDENTS OF RAM 1948-1965 SFE EDB 1689

RAIL ACCICENT, COTULLA, TEXAS - $12 / 1 / 73$

RAIL ACCICENT. INDIO, CALIFDRNIA - 6/25/73

RAIL ACCIDENT, SALEM, ILLINOIS - 6/10/71

RAIL ACCIDENT, MAQUIN, ILLINCIS - 5/24/72

RAIL ACCIDENT, TAFT, LOUISIANA - 2/21/73

RAIL ACCICENT, EAST ST LOUIS, ILLINOIS - $1 / 22 / 72$

RAIL ACCICENT, HOUSTON, TEXAS - 10/19/71

RAIL ACCIDENT, HERNDON, PENNSYLVANIA - 3/12/72

FREIGHT TRAIN ACCIDENTS, NUMEER OF CARS INVOLVET,

RAIL ACCIDENT - DNEONTA, NY - 2/12/74
UNC $\quad 01673-031+$

UNC $01675-036+$

UNC $01700-011+$

UNC $\quad 01701-001+$

UNC $\quad 01702-001+$

UNC $\quad 01703-002+$

UNC $01704-001+$

UNC $\quad 21705-006+$

UNC $\quad 21705-003+$

UNC 01708-001+

UNC $91709-003+$

UNC $\quad 21729-007+$

UNC $\quad 11782-004+$

...... TEMPERATURE ......... INPUT.... TKANSPURTATIGN.......... SHIP........

FIRF TFMPERATURES-SHIP UNC 00521-001+

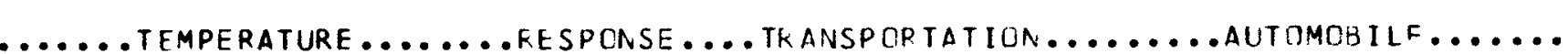

CARGO RESPONSF TRUCK AND RAIL ACCICENTS UNC O1688-0C2+ TRANSPORT ACCIDENTS OF RAM 194E TO 1965-UNITED KINGCOM UNC OI689-011+

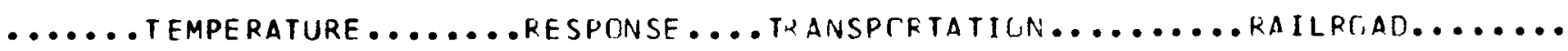

CARGO RESPGNSE TRUCK AND RAIL ACCCIDENTS SEE FDBIGBR

UNC $\quad 01699-002+$ ........WIND ........... INPUT .... TRANSPCRTATI .......AIRCRAFT......

HEL ICOPTER DOWNWASH CATA

UNC $\quad 21760-001+$

$\ldots \ldots \ldots \ldots . .$. ... ............ INPUT........GFNEPAL...........THEK.......

TCKNADCIS, FREQUENCY AAD LCCATIOA - UNITFL STATES

UNC $\quad 01541-011+$

WIND AND WAVE HEIGHTS - TROPICAL STORMS

TYPHCON REPORT - PACIFIC CLEAA - 1959

TYPHOOR PEPDRT - PACIFIC LCEAN - 1960

TYPHOON REPOPT - PACIFIC CCEAN - $19 t$

TYPHONN REPURT - PACIFIC CCEAN - 1962

TYPHOON REPORT - PACIFIC CCEAN - 19t3

IYPHUUN REPOPT - PACIFIC (CEAN - 1965

TYPHOON RFPORT - PACIFIC CCEAR - 1966

TYPHOON REPORT - PACIFIC ICEAR - 1067

TYPHOUN REPORT - PACIFIC OCEAR - 1S6O

TYPHOON REPORT - PACIFIC CCEAN - 1960

TYPHOON REPCPT - PACIFIC C'EAN - 107C

TYPHOON REPIRT - PACIFIC CCEAN - 1574

$\begin{array}{ll}\text { UNC } & 01636-002+ \\ \text { UNC } & 01802-003+ \\ \text { UNC } & 01803-003+ \\ \text { UNC } & 01804-003+ \\ \text { UNC } & 01805-004+ \\ \text { UNC } & 01806-003+ \\ \text { JNC } & 01307-003+ \\ \text { UNC } & 01868-003+ \\ \text { UNC } & 01809-003+ \\ \text { UNC } & 01810-004+ \\ \text { UNC } & 01811-002+ \\ \text { UNC } & 11812-003+ \\ \text { INNC } & 01813-003+\end{array}$


DISTRIBUTION :

TID-4500, UC-71 (124)

Transportation Branch (50)

ERDA

Division of Waste Management and Transportation Washington, DC 20545

National Science Foundation

2101 Constitution Avenue, NW

Washington, DC 20418

Attn: W. M. Carey

A. Grella (3)

DOT

Office of Hazardous Materials

Room 6218

Trans Point Building

Washington, DC 20590

L. Benner

Chief

Hazardous Materials Safety Division

NTSB

Washington, DC 20594

J. Russell

Environmental Protection Agency

Office of Radiation Programs

401 M Street, SW

Washington, DC 20460

W. F. Black

DOT

Federal Rall Administration

Trans Point Bullding

Washington, DC 20590

J. Buchanan

Nuclear Safety Infor mation Center

P. O. Box Y

Oak Ridge, TN 37830

E. E. Kidwell

DOT

Federal Highway Administration

DOT Building

Room 3408

Washington, DC 20590

W. E. Scott

National Highway Traffic Safety Administration

Office of Accident Investigation and Data

Analysis

Nassif Bullding

4007 th Street, SW

Washington, DC 20590

1000 G. A. Fowler

1200 W. A. Gardner

1500 R. L. Peurifoy, Jr.

1540 T. B. Lane

1541 S. W. Key

1542 C. A. Davidson

1542 J. T. Foley

1542 W. F. Hartman

1542 T. G. Priddy
H. C. Hardee

R. K. Clarke

D. W. Larson

R. T. Othmer

O. E. Jones

V. E. Blake, Jr.

M. R. Madsen

J. T. Risse

E. I. Bruce

A. W. Snyder

R. M. Jefferson

E. A. Aas (2)

L. S. Ostrander (5)

W. L. Garner (3)

For ERDA/TIC (Unlımited Release) 